\title{
Wavefield Decomposition-Based Direct Envelope Inversion and Structure-Guided Perturbation Decomposition for Salt Building
}

\author{
Pan Zhang ${ }^{1,2}$, Liguo Han ${ }^{1}$, Fengjiao Zhang ${ }^{1, *(\mathbb{D}}$, Qiang Feng ${ }^{1}$ and Xue Chen ${ }^{3}$ \\ 1 College of Geo-Exploration Science and Technology, Jilin University, Changchun 130026, China; \\ zhangpan@jlu.edu.cn (P.Z.); hanliguo@jlu.edu.cn (L.H.); fqfengq@163.com (Q.F.) \\ 2 Key Laboratory of Deep-Earth Dynamics of Ministry of Natural Resources, Institute of Geology, \\ Chinese Academy of Geological Sciences, Beijing 100037, China \\ 3 Hebei Key Laboratory of Strategic Critical Mineral Resources, Hebei GEO University, \\ Shijiazhuang 050031, China; cx3141@163.com \\ * Correspondence: zhangfengjiao@jlu.edu.cn; Tel.: +86-15504312747
}

Citation: Zhang, P.; Han, L.; Zhang,

F.; Feng, Q.; Chen, X. Wavefield

Decomposition-Based Direct

Envelope Inversion and

Structure-Guided Perturbation

Decomposition for Salt Building.

Minerals 2021, 11, 919. https://

doi.org/10.3390/min11090919

Academic Editor: Stanisław Mazur

Received: 17 July 2021

Accepted: 24 August 2021

Published: 25 August 2021

Publisher's Note: MDPI stays neutral with regard to jurisdictional claims in published maps and institutional affiliations.

Copyright: (C) 2021 by the authors. Licensee MDPI, Basel, Switzerland. This article is an open access article distributed under the terms and conditions of the Creative Commons Attribution (CC BY) license (https:// creativecommons.org/licenses/by/ $4.0 /)$.

\begin{abstract}
Due to the large-scale and strong perturbation features of salt bodies, it is very difficult to complete a good salt building with the conventional full waveform inversion (FWI) method without low-frequency data and prior information. The direct envelope inversion (DEI) method is quite effective for salt building when seismic data lack low-frequency information. However, in the current DEI studies, the calculation of the envelope field, which needs a nonlinear envelope operator, does not consider the influences of wavefield overlapping, and the inversion quality of subsalt areas needs further improvements. In this paper, we analyze the effects of wavefield overlapping on envelope field calculation and propose a new envelope field calculation method based on wavefield decomposition. Then, we propose a wavefield decomposition-based direct envelope inversion (WDDEI) method, in which the gradient is calculated using the new envelope field. To improve the inversion quality of subsalt structures, we propose a structure-guided perturbation decomposition method, which can separate the strong scattering salt information from the DEI results with the help of reverse time migration images. Finally, numerical tests are conducted on a modified SEG/EAGE salt model to demonstrate the effectiveness and the antinoise performance of the proposed method.
\end{abstract}

Keywords: full waveform inversion; direct envelope inversion; salt building; wavefield decomposition; perturbation decomposition

\section{Introduction}

Salt domes have been considered as good caprocks of oil and gas resources; therefore, high-precision salt building is attracting more and more attention in the field of exploration geophysics. Full waveform inversion (FWI) is the highest accuracy velocity reconstruction method for pre-stack seismic data [1]. However, it is difficult for conventional FWI to conduct good salt building in real cases because the large-scale and strong perturbation features of the salt body require abundant low-frequency data, which are always difficult to acquire. To reconstruct the salt velocity model in the absence of low-frequency data, many researchers modify the algorithm of conventional FWI and propose some promising methods. Laplace transformation of seismic signals can reconstruct sufficient low-frequency components, which is helpful for the recovery of the low-wavenumber structures of salt bodies. Based on this, Laplace domain waveform inversion [2] and Laplace-Fourier domain waveform inversion [3] are proposed. It is difficult for conventional FWI to update salt geometry due to strong scattering properties. Combining the level-set approach with the FWI workflow has proven to be a promising method to effectively build and modify the salt boundaries [4,5]. The advantages of total variation constraint can also help the reconstruction of salt structures. The total variation is a sparse constraint, which 
minimizes the gradient of model parameters. Total variation-constrained FWI can provide sharp boundaries and constant velocity inside the salt body [6-8]. There are also research studies that compensate for the loss of low-frequency information using special low-wavenumber-retrieving-based FWI $[9,10]$. Some researchers try to solve problems concerning salt building by combining deep learning methods with FWI, which has also made good progress [11,12].

The direct envelope inversion (DEI) method has been proven to be a quite effective method for salt building in the case of a lack of low-frequency information [13,14]. Different from the conventional envelope inversion method [15-17], the DEI method defines a direct envelope Fréchet derivative, which can directly map the low-frequency envelope residuals to the large-scale strong scattering velocity perturbations. Some researchers have developed the original DEI method since it was first proposed. To improve the inversion quality of salt bodies and the subsalt structures, the reflection DEI method has been proposed [18]. The original DEI method is sensitive to source wavelet errors. The source-independent DEI method can mitigate the effects of inaccurate source wavelets on final inversion results [19]. The envelope calculated by Hilbert transformation loses polarity information. The signed DEI method makes up for this disadvantage and improves the inversion quality of deep structures by adding polarity to envelope calculation [20]. Other improvements of the DEI method include multi-offset DEI strategy [21], joint instantaneous amplitude and phase DEI [22], and angle domain DEI [23]. In the above DEI methods, the calculation algorithm of the envelope field can be classified into two categories. One is calculating the envelope field with the help of Hilbert transformation, which will lead to the absence of polarity information. The other is calculating the envelope field by the nonlinear interpolation method, which keeps some polarity information but does not have explicit expression. However, all the methods mentioned above do not consider the effects of wavefield overlapping on neither the envelope field calculation nor the DEI process.

In this paper, we analyze the effects of wavefield overlapping on the envelope field calculation and propose a new envelope field calculation method based on wavefield decomposition. Then, we propose a wavefield decomposition-based direct envelope inversion (WDDEI) method, in which the gradient is calculated using the new envelope field. To improve the inversion of subsalt structures, we propose a structure-guided perturbation decomposition method, which can separate the strong scattering salt information from the DEI results with the help of reverse time migration (RTM) images. Finally, numerical tests are conducted on a modified SEG/EAGE (Society of Exploration Geophysicists/European Association of Geoscientists \& Engineers) salt model to demonstrate the effectiveness and the antinoise performance of the proposed method.

\section{Review of Full Waveform Inversion, Conventional Envelope Inversion and Direct Envelope Inversion}

2.1. Conventional Full Waveform Inversion and Envelope Inversion

The objective function of conventional FWI can be written as [24]

$$
\sigma_{F W I}=\frac{1}{2} \sum_{s r} \int_{0}^{T}\left[d_{s y n}(t)-d_{o b s}(t)\right]^{2} d t,
$$

where $d_{\text {syn }}$ and $d_{\text {obs }}$ denote the synthetic and observed seismic data, respectively; sr denotes all the source and receiver position; $t$ denotes time; $T$ denotes total recording time length. The derivative of $\sigma_{F W I}$ with respect to velocity $v$ can be calculated as

$$
\frac{\partial \sigma_{F W I}}{\partial v}=\sum_{s r} \int_{0}^{T} \frac{\partial d_{s y n}(t)}{\partial v}\left[d_{s y n}(t)-d_{o b s}(t)\right] d t,
$$

where $\frac{\partial d_{s y n}(t)}{\partial v}$ is the sensitivity kernel (the Fréchet derivative). A problem for FWI is that it may suffer from the "cycle-skipping" problem if seismic data lack low-frequency information. 
Wu et al. [16] propose taking full advantage of the ultra-low-frequency information embedded in the envelope data to mitigate the cycle-skipping problem of FWI. The envelope data are also proven to contain the intrinsic long-wavelength responses of the subsurface media. The objective function of the conventional envelope inversion (EI) can be written as [16]

$$
\sigma_{E I}=\frac{1}{2} \sum_{s r} \int_{0}^{T}\left[e_{s y n}(t)-e_{o b s}(t)\right]^{2} d t,
$$

where $e_{s y n}(t)$ and $e_{o b s}(t)$ denote the synthetic and observed envelope data, respectively. The synthetic envelope data can be calculated as

$$
e_{s y n}=\sqrt{d_{s y n}^{2}+\left[H\left(d_{s y n}\right)\right]^{2}}
$$

where $H(\cdot)$ denotes the Hilbert transform. The observed envelope data $e_{o b s}$ can be calculated in a similar way.

The derivative of $\sigma_{E I}$ with respect to velocity can be written as

$$
\frac{\partial \sigma_{E I}}{\partial v}=\sum_{s r} \int_{0}^{T} \frac{\partial d_{s y n}(t)}{\partial v}\left[\frac{\Delta E d_{s y n}}{e_{s y n}}-H\left\{\frac{\Delta E d_{s y n}^{H}}{e_{s y n}}\right\}\right] d t,
$$

where $\Delta E$ is the envelope data residual, and $d_{s y n}^{H}$ denotes the Hilbert transform of the synthetic seismic data. The derivation of Equation (5) uses the chain rule, so it has the same Fréchet derivative $\frac{\partial d_{s y n}(t)}{\partial v}$ as conventional FWI. In other words, the conventional EI method does not completely solve the problems of FWI. It cannot handle the inversion in strong scattering cases either.

\subsection{Direct Envelope Inversion}

The difficulty of using FWI for salt building is mainly that the target areas always relate to large-scale and strong velocity perturbations, which require ultra-low-frequency information to reconstruct large-scale salt bodies. However, real seismic data always lack effective low-frequency information or have very low SNR in the low-frequency band. Therefore, it is very difficult for conventional FWI to solve salt building problems in real conditions. The DEI method has proven to be an effective method for salt building, even without low-frequency data [14]. The objective function for DEI $\sigma_{D E I}$ has the same expression with $\sigma_{E I}$ as Equation (3). However, in the DEI method, the derivative of $\sigma_{D E I}$ with respect to velocity $v$ can be written as

$$
\frac{\partial \sigma_{D E I}}{\partial v}=\sum_{s r} \int_{0}^{T}\left(\frac{\partial e_{s y n}}{\partial v}\right)^{T}\left(e_{s y n}-e_{o b s}\right) d t
$$

where $\frac{\partial e_{s y n}}{\partial v}$ is defined as the direct envelope Fréchet derivative, which can be written as

$$
\frac{\partial e_{s y n}}{\partial v}=\mathbf{G}_{0}^{(e)} \mathbf{Q}_{0}^{(e)}
$$

where $\mathbf{G}_{0}^{(e)}$ is the envelope Green function, and $\mathbf{Q}_{0}^{(e)}$ is the envelope virtual source operator. From Equation (6), we know that the envelope residual is directly mapped to the velocity perturbation with the help of the direct envelope Fréchet derivative. Therefore, the ultralow-frequency information contained in the envelope residuals can be directly used to recover the low-wavenumber components of the velocity perturbations.

The direct envelope inversion method is good at solving situations where largescale salt domes exist in the target area, and does not depend on the ultra-low-frequency information in the original seismic data, which are not well solved by conventional FWI. The disadvantage of this method is that it can only recover the large-scale salt body 
information, but cannot perform an effective inversion of the detailed structures. This part should depend on FWI.

\section{Wavefield Decomposition-Based Direct Envelope Inversion}

\subsection{Envelope Field Calculation Based on Wavefield Decomposition}

In the DEI method, we need envelope Green's function $\mathrm{G}_{0}^{(e)}$ to describe the intrinsic reflection responses of the incident energy pulse. However, currently, we have not found a way to calculate the $\mathbf{G}_{0}^{(e)}$ explicitly. Therefore, the envelope field is approximately calculated by taking the envelope of the 3D time-space domain wavefield. This approximation is helpful for realizing the DEI algorithm. However, as we know, the envelope operator is a nonlinear operator, which does not satisfy the superposition principle. Therefore, it may cause obvious errors due to the overlapping of wavefields. In particular, this will result in some interference during the inversion, making it difficult to achieve uniformity inside the salt body. We propose calculating the envelope field based on the wavefield decomposition, which can correct the overlapping errors of the envelope field and suppress part of the interference to make the salt body more uniform.

Assume two events are overlapped at a certain time point. The wavefield amplitudes of the two events at the overlapping point are $y_{1}$ and $y_{2}$, respectively. The envelope value at the overlapping point can be calculated as

$$
e_{o v l}=\sqrt{\left(y_{1}+y_{2}\right)^{2}+\left[H\left(y_{1}+y_{2}\right)\right]^{2}} .
$$

If we consider envelope propagation, the true envelope value at the overlapping point should be

$$
e_{\text {prop }}=e_{1}+e_{2}
$$

where $e_{1}$ and $e_{2}$ are envelope values of two independent events. Since $e_{1}$ and $e_{2}$ can be independently calculated by Equation (4), Equation (9) can be written as

$$
e_{\text {prop }}=\sqrt{y_{1}^{2}+\left[H\left(y_{1}\right)\right]^{2}}+\sqrt{y_{2}^{2}+\left[H\left(y_{2}\right)\right]^{2}} .
$$

Obviously, $e_{\text {ovl }}$ does not equal to $e_{\text {prop }}$. Therefore, in the overlapping point, the previous calculation methods for the envelope Green function will cause some errors.

To deal with this kind of error, we should carefully process the envelope calculation at overlapping regions. According to the nonlinearity of the envelope operator shown in Equations (8) and (10), we propose conducting wavefield decomposition before calculating the envelope field. Assuming the forward propagating wavefield is $P_{f}$, we first decompose it into upgoing wavefield $P_{f-u p}$ and downgoing wavefield $P_{f-\text { down }}$ as

$$
P_{f}=P_{f-u p}+P_{f-\text { down }}
$$

The wavefield decomposition can be conducted by a 2D fast Fourier transform [25]. Then, we decompose $P_{f-u p}$ and $P_{f-\text { down }}$ into leftgoing and rightgoing wavefields, respectively. Therefore, Equation (11) can be written as

$$
P_{f}=P_{f-u p-l e f t}+P_{f-u p-r i g h t}+P_{f-\text { down-left }}+P_{f-\text { down-right }} .
$$

Taking the envelopes of $P_{f-u p-l e f t}, P_{f-u p-r i g h t}, P_{f-\text { down-left }}$ and $P_{f-\text { down-right }}$, respectively, and conducting additions, we can obtain an accurate forward propagating envelope field as

$$
P_{f-w d}^{(e)}=P_{f-u p-l e f t}^{(e)}+P_{f-u p-r i g h t}^{(e)}+P_{f-\text { down-left }}^{(e)}+P_{f-\text { down-right }}^{(e)} .
$$

where $P_{f-u p-l e f t}^{(e)}, P_{f-u p-r i g h t}^{(e)}, P_{f-\text { down-left }}^{(e)}$ and $P_{f-\text { down-right }}^{(e)}$ are the envelope field of $P_{f-u p-l e f t}, P_{f-u p-r i g h t}, P_{f-\text { down-left }}$ and $P_{f-\text { down-right }}$, respectively. 
We conduct a numerical test to compare the envelope field calculated by the conventional method and the proposed method. Figure $1 \mathrm{a}$ is the velocity model we use. Figure $1 \mathrm{~b}$ shows a snapshot of the wavefield at $1 \mathrm{~s}$. It is obvious that the reflected waves and diffracted waves are overlapped at this time point. The envelope field calculated by the conventional method is shown in Figure 1c, and the envelope field calculated by the proposed method is shown in Figure 1d. Comparing the events in the red rectangle areas, we can see that the envelope field calculated by the proposed method better reflects the macro-information of the fluctuation.
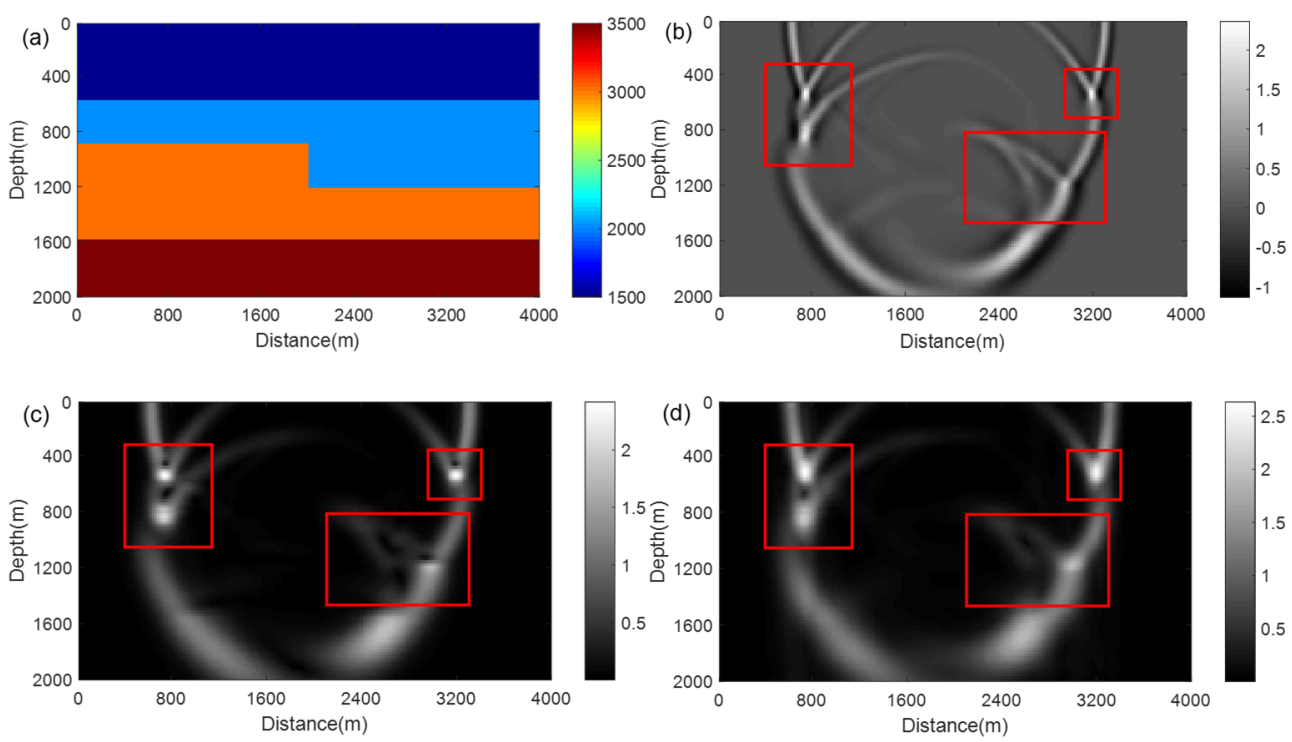

Figure 1. Envelope field calculated by different methods. (a) Velocity model. (b) Wavefield snapScheme 1 s. (c) Envelope field calculated by conventional method at $1 \mathrm{~s}$. (d) Envelope field calculated by the proposed method at $1 \mathrm{~s}$. Red rectangulars show the comparison areas.

\subsection{Wavefield Decomposition Based Direct Envelope Inversion}

From the above analysis, we know that the envelope field calculation method based on wavefield decomposition is more accurate than the previous methods. We replace the envelope field calculation process in DEI with the proposed method and obtain a new inversion method called the wavefield decomposition-based direct envelope inversion (WDDEI) method. The objective function of WDDEI is the same as that of DEI, which is shown in Equation (3).

From Equations (6) and (7), we know that the gradient of DEI can be calculated by the adjoint-state method. The detailed calculation of $\mathbf{G}_{0}^{(e)}$ and $\mathbf{Q}_{0}^{(e)}$ in Equation (7) can be found in Reference [19]. According to the adjoint-state method [26], Equation (6) can be calculated by the zero-lag cross-correlation of the forward-propagating envelope field $P_{f}^{(e)}$ and back-propagating residual field $P_{b}^{(e)}$ as

$$
\frac{\partial \sigma_{D E I}}{\partial v}=\frac{1}{v_{0}} P_{f}^{(e)} P_{b}^{(e)}
$$

where $v_{0}$ is the background velocity. When considering wavefield decomposition, Equation (6) can be modified as

$$
\frac{\partial \sigma_{I D E I}}{\partial v}=\sum_{s r} \int_{0}^{T}\left(\frac{\partial\left(\sum_{\theta} e_{s y n}\right)}{\partial v}\right)^{T}\left(e_{s y n}-e_{o b s}\right) d t
$$

where $\sigma_{I D E I}$ denotes the objective function of WDDEI, and $\theta$ denotes the wave propagated angle after wavefield decomposition. The derivation of Equation (15) is similar 
to the derivation of Equation (6). Substituting the forward envelope field calculated by Equation (13) into (14), we can obtain the gradient formula of WDDEI as

$$
\frac{\partial \sigma_{I D E I}}{\partial v}=\frac{1}{v_{0}} P_{f-w d}^{(e)} P_{b}^{(e)},
$$

In the current WDDEI method, $P_{b}^{(e)}$ is not decomposed. This means we calculate the envelope with wavefield decomposition only for the virtual source, and we do not apply wavefield decomposition when we calculate the back-propagated wavefields. There are two reasons why we do not need wavefield decomposition for the back-propagated wavefields. On the one hand, in the implementation of the DEI method, we calculate the forward-propagated envelope fields by taking the envelope of forward-propagated wavefields. Therefore, we can conduct wavefield decomposition before calculating the envelope fields to overcome the effects of wavefield overlapping. However, in the calculation of back-propagated envelope fields, the adjoint source is the envelope residual, but not the waveform residual. The wave equation can propagate relatively accurate amplitude information about the envelope field [19]. Thus, we do not need wavefield decomposition in this step. This is also an approximation of this method, which cannot be solved by the wavefield decomposition strategy. On the other hand, if we back-propagate the waveform residual, and then, take the envelope to generate the back-propagated envelope fields, the polarity of the wavefield will be missing because the forward-propagated envelope field lacks polarity information. If there is no polarity information in the forward-and back-propagated envelope fields, the inversion quality will be greatly affected. The polarity information retained in the back-propagated envelope fields can help to recover the bottom of the salt body. The workflow of the proposed WDDEI method is shown in Figure 2.

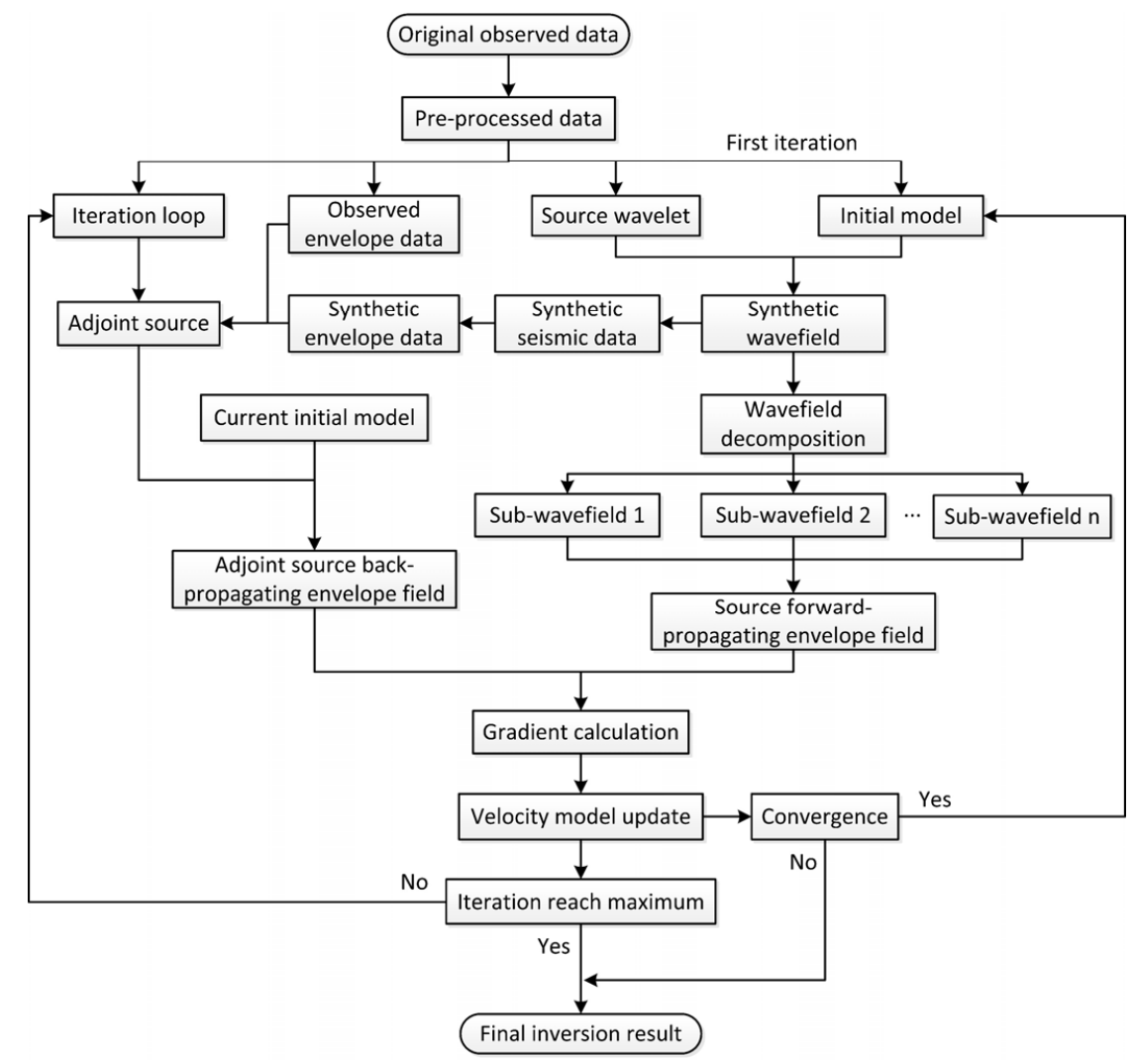

Figure 2. The workflow of the proposed WDDEI method. 


\section{Structure-Guided Perturbation Decomposition (SGPD)}

WDDEI can help to reconstruct the velocity of large-scale salt bodies. The FWI calculation based on WDDEI results can recover the weak scattering structures around the salt bodies. However, the resolution of WDDEI results is relatively low. The inverted salt boundaries, especially the bottom boundary, are blurry. Since the inversion of strong scattering structures is difficult for FWI, the subsalt areas always have spurious energy after the WDDEI + FWI process if the boundary of the salt body is not accurately determined. Therefore, we need a new strategy to help correct the boundaries of the salt bodies and improve the subsalt inversion. The perturbation decomposition strategy effectively decomposes weak and strong perturbations during the inversion process [19]. If we keep the strong perturbation area unchanged and reinvert the weak perturbation area only, the high-velocity artifacts can be suppressed. To judge whether the perturbation at one grid point is strong or not, we can use the following definition of strong perturbations:

$$
\frac{\left|v(x, z)-v_{0}(x, z)\right|}{v_{0}(x, z)}>\alpha,
$$

where $v$ is the inverted velocity; $v_{0}$ is the initial velocity; $x$ and $z$ are the horizontal and vertical positions, respectively; $\alpha$ is the perturbation factor. According to our experience, $\alpha$ larger or equal to 0.9 is a good indicator of strong perturbations for a general salt model. In the subsalt area of the inversion results, there are always high-velocity artifacts. The choice of $\alpha$ is important and difficult if we have no prior information. A large $\alpha$ may damage the salt structures, and a small $\alpha$ may leave spurious energies in the inversion result.

In previous studies of perturbation decomposition, the choice of $\alpha$ value is mainly based on human experience, which is very difficult to determine because we assume we do not know any prior information about the salt body. To make it easier to choose a suitable $\alpha$ value, we propose a structure-guided perturbation decomposition strategy. We aim to control the $\alpha$ value by using RTM images, since the velocity model provided by WDDEI + FWI is usually highly accurate except for some high-velocity abnormalities near or below the salt body. RTM based on WDDEI + FWI results can easily image the boundaries of the salt body. In the artificial high-velocity area, the RTM results may not provide any reasonable structures. Therefore, we can choose a suitable $\alpha$ value according to the RTM results, which we call the structure-guided perturbation decomposition (SGPD) process. The inversion workflow with the SGPD strategy is shown in Figure 3. We conduct reverse time migration (RTM) using the velocity model of WDDEI + FWI. Then, we compare the velocity profile and the RTM image. At the salt dome position in the velocity profile, the RTM imaging profile can help to depict the salt dome boundaries. At the spurious energy position in the velocity profile, the RTM imaging profile does not indicate any salt structure. According to this principle, we can choose a suitable $\alpha$ to suppress the spurious energy but retain the salt dome information.

The application condition of the perturbation decomposition approach is that the background velocity excluding the salt dome is significantly different from that of the salt dome, and the velocity perturbations are within the range that FWI can handle. Therefore, reinversion can be performed by refilling the initial velocity into the background area. The advantage of this method is that it can effectively separate the strong and weak perturbation areas and optimally perform the inversion, respectively. The disadvantage is that if the background velocity perturbation exceeds the range FWI can handle, cycle-skipping will also occur during the inversion process. 


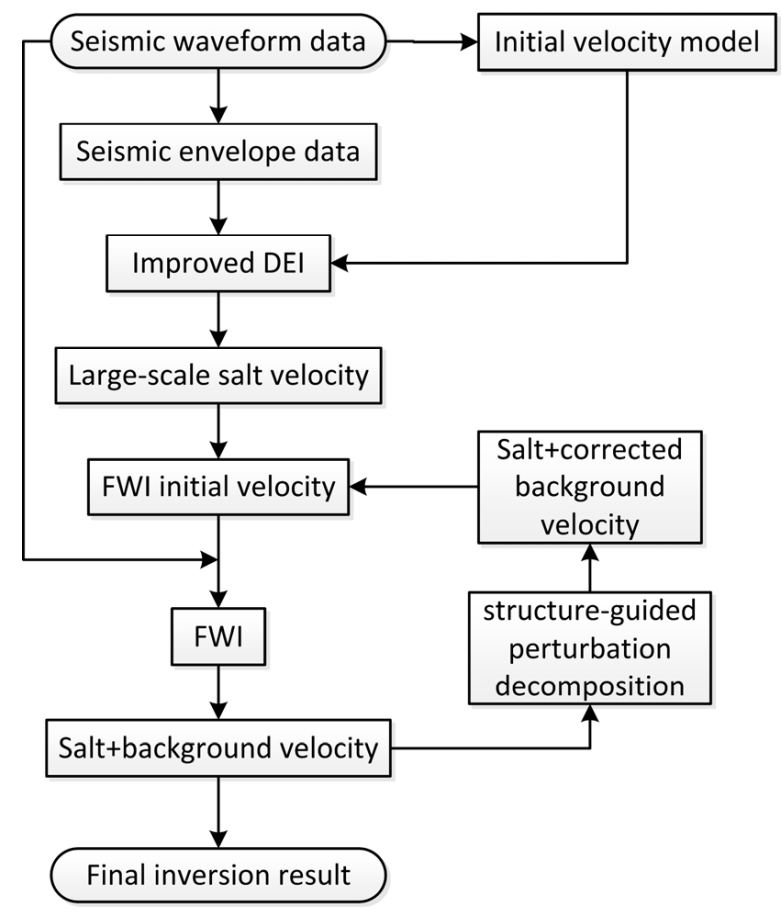

Figure 3. The workflow of SGPD inversion strategy.

\section{Numerical Examples}

In this section, we test the proposed method using a modified SEG/EAGE salt model. The SEG/EAGE salt model is a typical model to test methods for salt building. The commonly used Marmousi model is a typical weak perturbation model that is suitable to test conventional FWI or EI methods. The sensitivity kernel used in this paper is only effective for a strong scattering case demonstrated by [14] in detail. Therefore, we do not conduct tests on the Marmousi model. The true velocity model is shown in Figure 4a. The background velocity is about $2500 \mathrm{~m} / \mathrm{s}$. At the top-center of the model, there is a high-velocity salt body with a velocity of $4482 \mathrm{~m} / \mathrm{s}$ and an average thickness of about $400 \mathrm{~m}$. Therefore, the salt dome is a typical strong scatter to the background velocity structures. The initial model is a linear gradient model, which only reflects the background velocity range without any salt information (Figure $4 b$ ). The source wavelet is a Ricker wavelet with a dominant frequency of $9 \mathrm{~Hz}$ without low-frequency information below $4 \mathrm{~Hz}$. The waveform and the frequency spectrum of the source wavelet are shown in Figure 5.

First, we test the inversion quality of the conventional DEI and FWI methods. Using Figure $4 \mathrm{~b}$ as the initial model, the conventional FWI result is shown in Figure 6a. It can only recover some top boundary information of the salt dome. The conventional DEI result using Figure $4 \mathrm{~b}$ as the initial model is shown in Figure $6 \mathrm{~b}$, which can reconstruct obvious large-scale salt information but with some shallow spurious velocity areas. The multiscale FWI results based on Figure $6 \mathrm{~b}$ are shown in Figure $6 c, d$, respectively. In the final inversion result (Figure 6d), the velocity inside the salt is not uniform and there are some errors in the subsalt area. Figure 6e is the difference profile between the true velocity model (Figure 4a) and the DEI + FWI result (Figure 6d). We can see that there are obvious residuals both inside the salt body and in the subsalt area. If the DEI process cannot recover parts of the strong perturbations accurately, the successive FWI process can hardly correct the strong velocity residuals.

Then, we test the inversion quality of the WDDEI method. Using Figure $4 \mathrm{~b}$ as the initial model, the WDDEI result is shown in Figure 7a. Compared with Figure 6b, we know that the WDDEI method can improve the shape and internal velocity inversion and suppress spurious energies in the shallow part. The multiscale FWI results based on Figure $7 \mathrm{a}$ are shown in Figure $7 \mathrm{~b}, \mathrm{c}$, respectively. We can see that the final inversion result 
(Figure 7c) is largely improved in the inversion of the salt body compared with Figure 6d. However, there are still some errors in the subsalt areas. Figure $7 d$ is the difference profile between the true velocity model (Figure 4a) and the WDDEI + FWI result (Figure 7c). Compared with Figure 6e, the velocity residual inside the salt body is much smaller in Figure $7 \mathrm{~d}$. However, in the subsalt area, there are strong velocity residuals (blue residuals in Figure 7d) that conventional FWI cannot correct.

Using Figure 7c as a velocity model, we conduct RTM. The result is shown in Figure 8a. Using Figure 8a as a structure constraint, the perturbation decomposition result is shown in Figure $8 \mathrm{~b}$. The salt body is successfully extracted, and the artifacts in the subsalt area are suppressed. The background velocity outside the salt body in Figure $8 \mathrm{~b}$ is the same as the original initial model (Figure 4 b). The FWI result using Figure 8 b as the initial model is shown in Figure 8c. We can see that both the salt body and many subsalt structures are effectively recovered. The final inversion result is much better than the conventional result (Figure $6 \mathrm{~d}$ ) both in the salt body and subsalt reconstruction. In the background velocity on both sides of the salt body, there are some low-frequency artifacts, which also exist in the conventional FWI result (Figure 6a). However, the artifacts in the final inversion result do not influence the accurate interpretation of the salt body and most of the fine structures. Figure $8 \mathrm{~d}$ is the difference profile between the true velocity model (Figure $4 \mathrm{a}$ ) and the SGPD result (Figure $8 b$ ). Compared with Figure $7 d$, we know that the strong velocity residuals (blue residuals) are suppressed after the perturbation decomposition process. Since the background velocity in Figure $8 b$ is the same as Figure $4 b$, there are mainly some relatively weak residuals outside the salt body in Figure 8d, which is much easier to handle by conventional FWI. Figure $8 \mathrm{e}$ is the difference profile between the true velocity model (Figure 4a) and the final inversion result (Figure 8c). Compared with Figure 8d, the velocity residuals in the shallow part and the salt boundaries are much weaker. The velocity residuals in the subsalt area in Figure 8e are much weaker than those in Figure 7d, which demonstrates that the proposed structure-guided perturbation decomposition strategy is helpful for improving the inversion quality in the subsalt area.
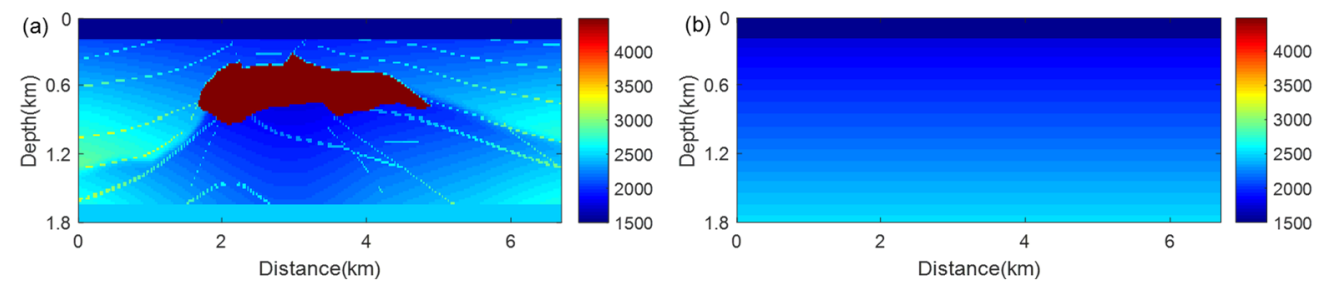

Figure 4. True and initial velocity model. (a) The true velocity model with a strong scattering salt body. (b) The initial velocity model without any salt information.
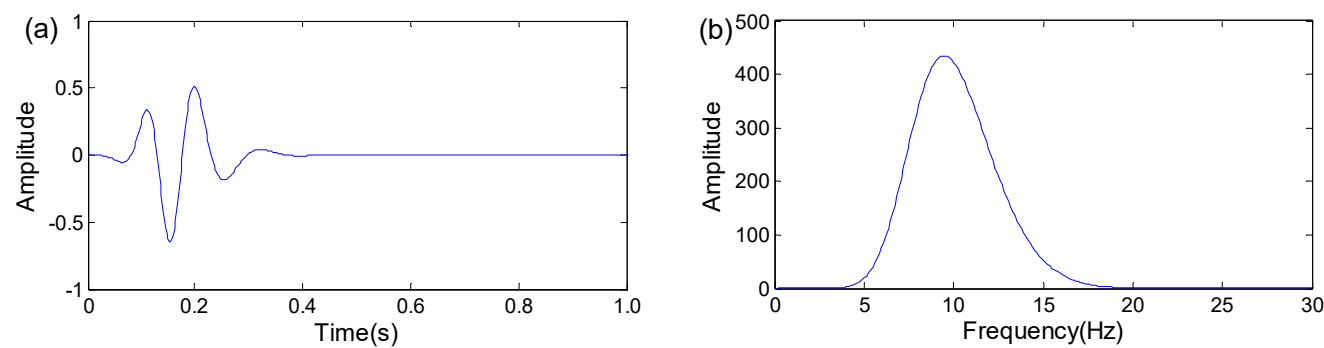

Figure 5. Source wavelet. (a) The waveform of the source wavelet. (b) The spectrum of source wavelet. 

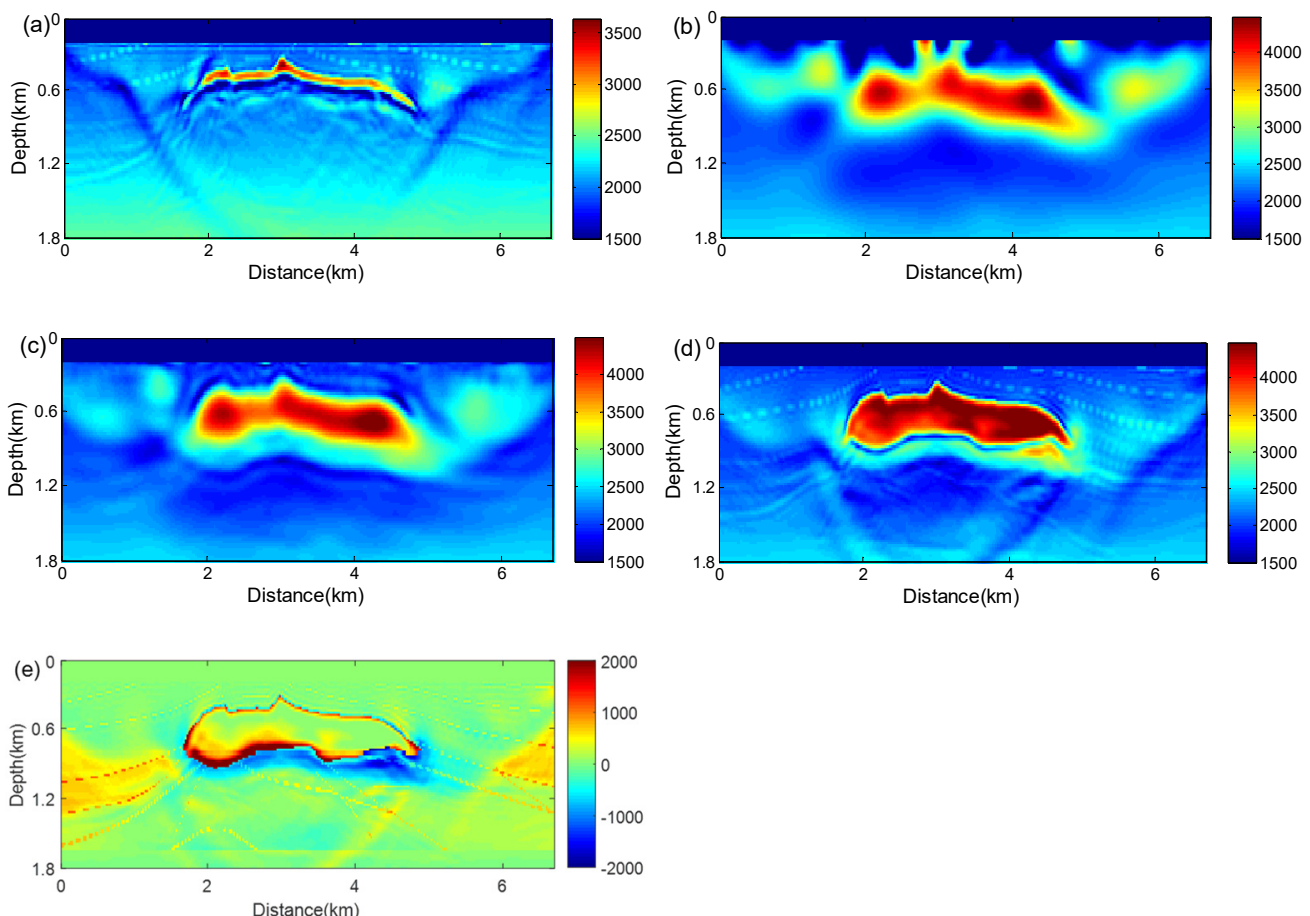

Figure 6. Inversion results by conventional DEI and FWI. (a) Conventional FWI result. (b) Conventional DEI result. (c) Conventional DEI + FWI result with data frequency band of 4-8 Hz. (d) Conventional DEI + FWI $(4-8 \mathrm{~Hz})+$ FWI $(4-18 \mathrm{~Hz})$ result. (e) Difference profile between Figures $4 \mathrm{a}$ and $6 \mathrm{~d}$.
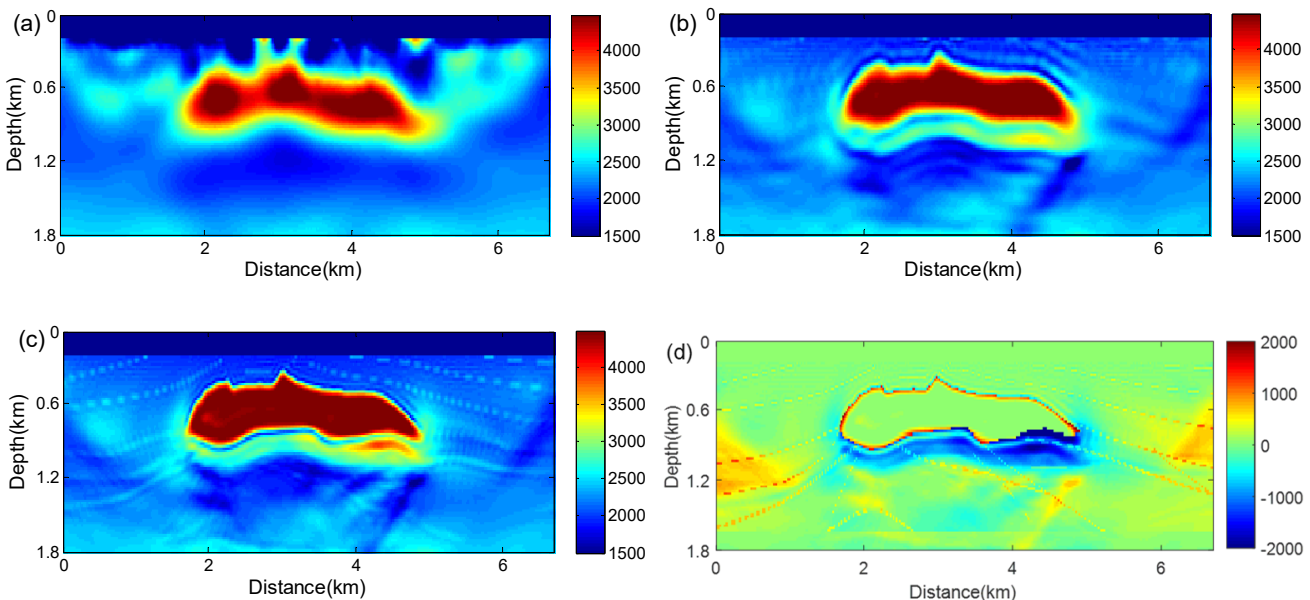

Figure 7. Inversion results by WDDEI and FWI. (a) WDDEI result. (b) WDDEI + FWI result with data frequency band of 4-8 Hz. (c) WDDEI + FWI (4-8 Hz) + FWI (4-18 Hz) result. (d) Difference profile between Figures $4 \mathrm{a}$ and $7 \mathrm{c}$.
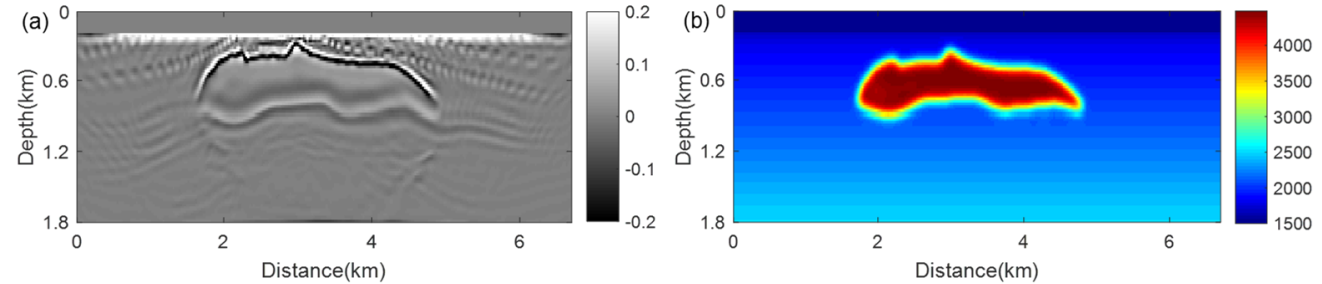

Figure 8. Cont. 

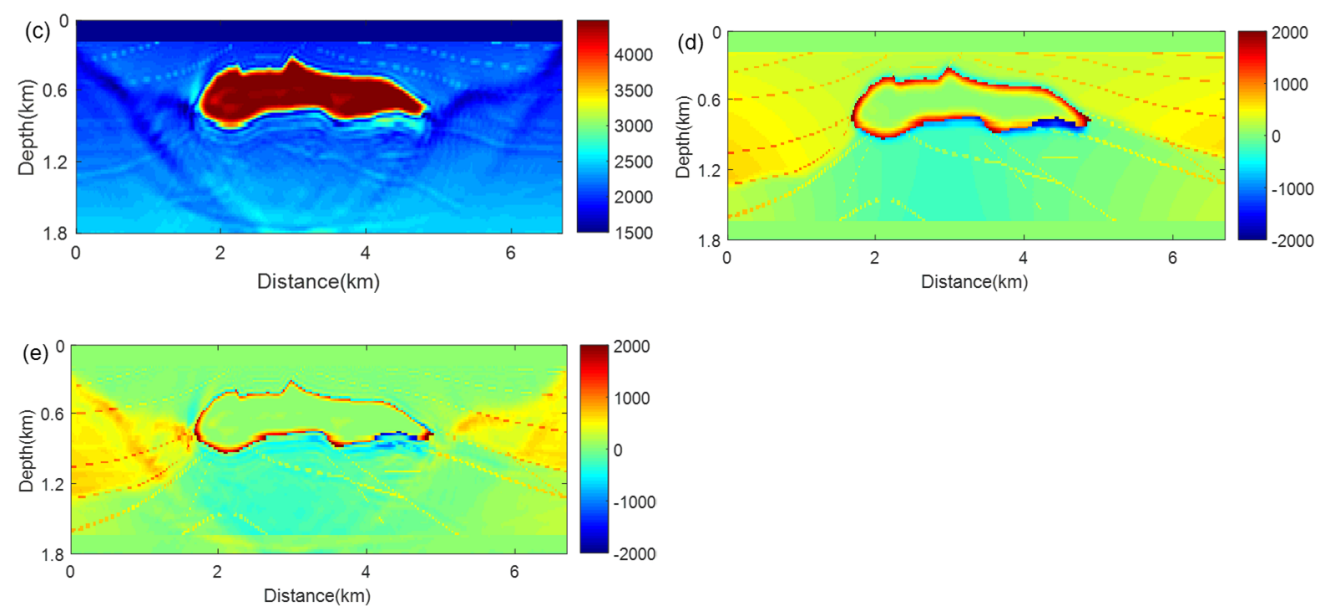

Figure 8. Inversion results by WDDEI and SGPD method. (a) RTM imaging result using Figure 7d as velocity model. (b) SGPD result. (c) Final inversion result of the proposed method. (d) Difference profile between Figures $4 \mathrm{a}$ and $8 \mathrm{~b}$. (e) Difference profile between Figures $4 \mathrm{a}$ and $8 \mathrm{c}$.

Finally, we test the antinoise performance of the proposed method. In the following test, all the simulation parameters are the same as the above tests. One of the single-shot records is shown in Figure 9a. White Gaussian noise with a signal to noise ratio of 20 is added to the original seismic records. The noisy data with the same source location of Figure 9a are shown in Figure 9b. We can see that many detailed reflection events are covered by noise. In the inversion tests, we still use the true velocity model and the initial velocity model shown in Figure 4a,b. The conventional DEI result is shown in Figure 10a. Compared with Figure $6 \mathrm{~b}$, we know that the noise influences the reconstruction of the salt body and also brings some shallow high-velocity errors. The DEI + FWI result is not as good as Figure 6d, especially inside the salt body. The WDDEI result is shown in Figure 10c. Although it is not as good as Figure 7a due to the interference of noise, the WDDEI result is better than the DEI result. The WDDEI + FWI result is shown in Figure 10d. Compared with Figure 10b, the inversion quality of the subsalt area in Figure 10d is not improved, but the velocity inside the salt body is more uniform. For the subsalt velocity inversion, we can use the proposed SGPD approach. The RTM image, using Figure 10d as a velocity model, is shown in Figure 10e. Using it as a structure control, we can choose a suitable $\alpha$ value, which is 0.8 . The perturbation decomposition result is shown in Figure 10f. We can see that the large-scale salt body is extracted, and the background artifacts are suppressed. The final inversion result is shown in Figure 10. We know that the proposed method can recover relatively accurate salt structures and background structures with a moderate noise level, although the velocity inside the salt body is not as uniform as the noise-free case.

To further compare the inversion results, we calculate some difference profiles in Figure 11. The difference profiles between the true velocity model and the DEI + FWI result, the WDDEI + FWI result, the perturbation decomposition result, and the final inversion result are shown in Figure 11a-d, respectively. We can see that in the test noise level, the proposed approach can still show obvious advantages compared with the conventional inversion workflow. 

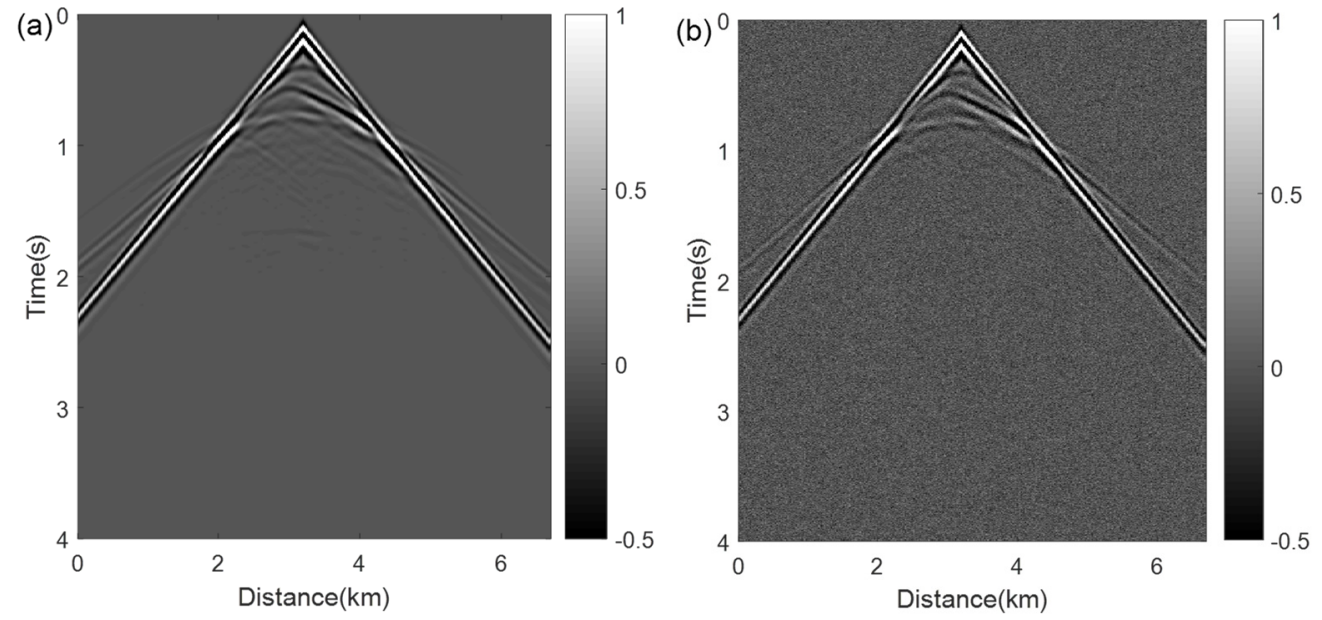

Figure 9. Noisy data used in this test. (a) Noise-free data. (b) Noisy data.
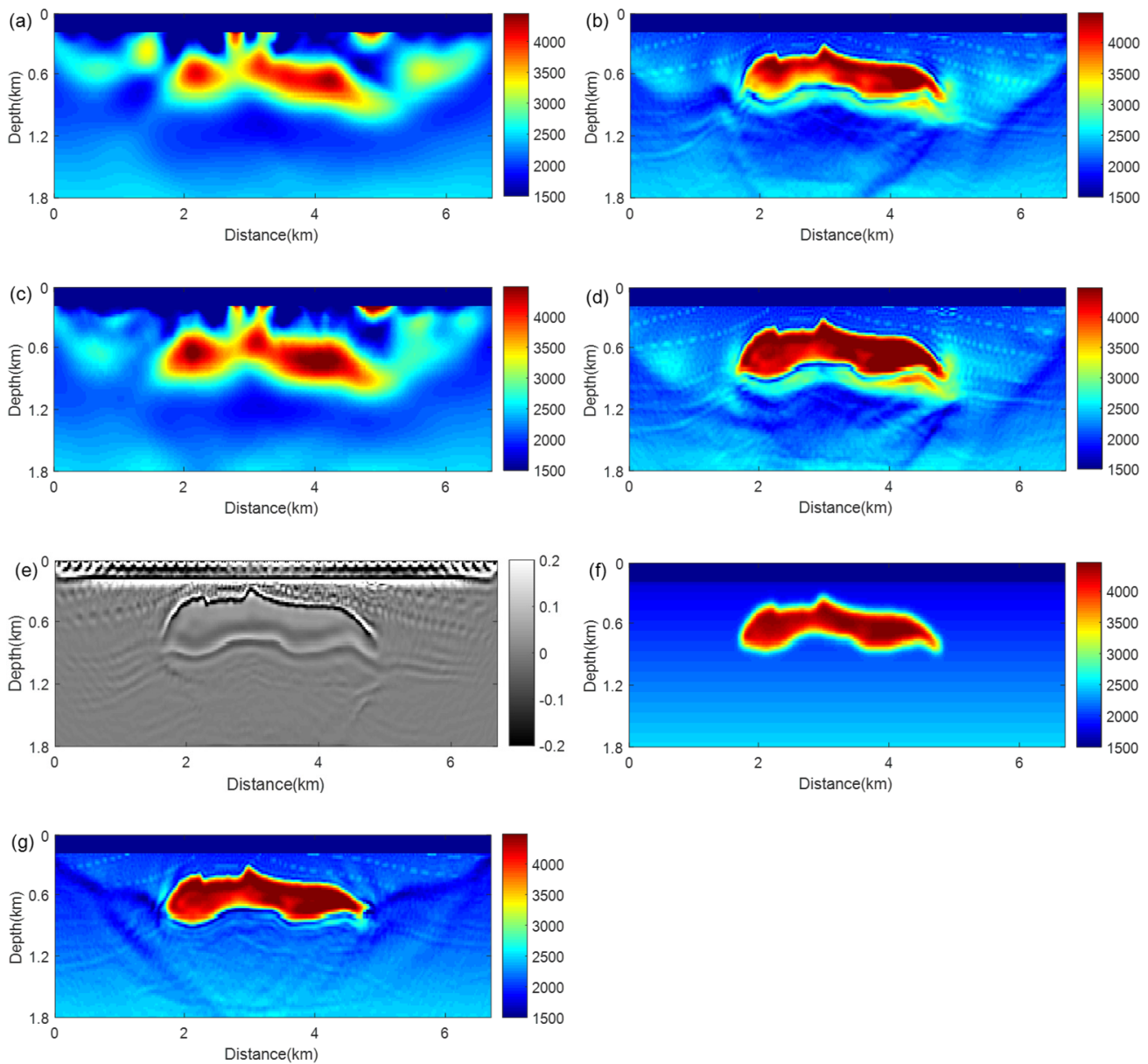

Figure 10. Inversion results with noisy data. (a) Conventional DEI result. (b) Conventional DEI + FWI result. (c) WDDEI result. (d) WDDEI + FWI result. (e) RTM imaging result using Figure 10d as the velocity model. (f) SGPD result. (g) Final inversion result of the proposed method. 

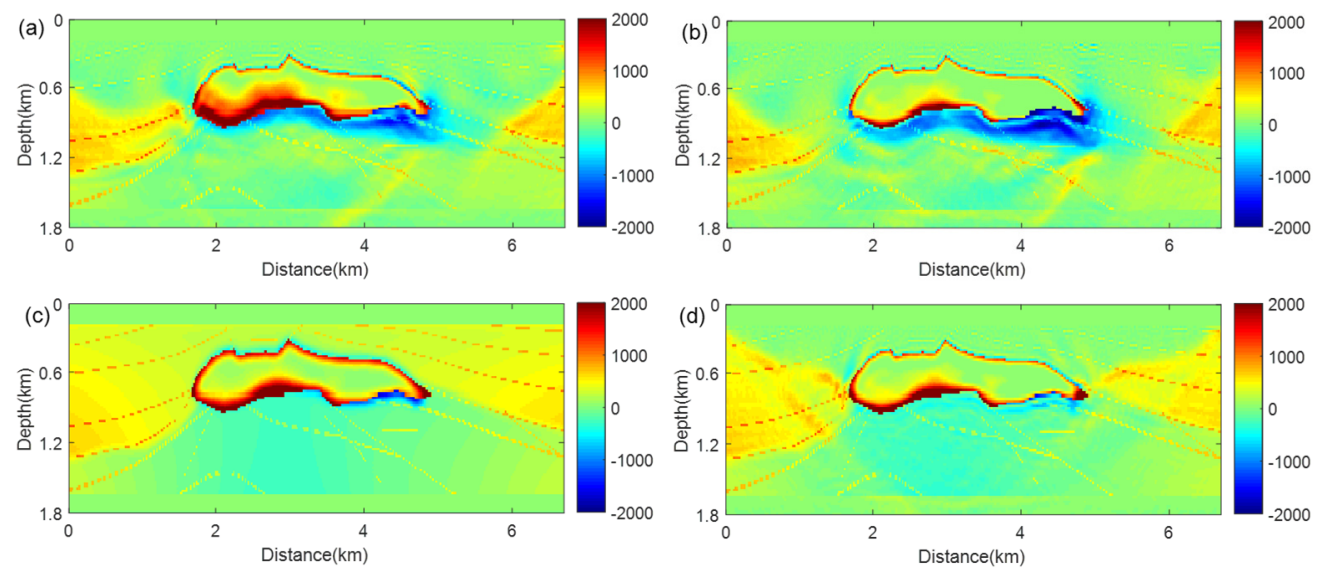

Figure 11. Difference profiles. (a) Difference profile between Figures $4 \mathrm{a}$ and 10b. (b) Difference profile between Figures 4a and 10d. (c) Difference profile between Figures 4a and 10f. (d) Difference profile between Figures $4 \mathrm{a}$ and $10 \mathrm{~g}$.

\section{Discussion and Conclusions}

From the theoretical analysis in Section 3.1, we know that our method effectively overcame the wavefield overlapping effects when the propagations of the incident and reflected wavefields are in opposite directions. To make up for this defect as much as possible, we propose decomposition of the wavefields into four directions, i.e., up-left, up-right, down-left, and down-right directions. However, if the wavefields cannot be separated into these four directions, overlapping effects will exist and influence the final inversion results. In our studies, we believe that we have already met this problem. From the inversion results shown in this paper, we know that this kind of error may have little influence on the final inversion results. For more complex models and applications, we think introducing more detailed wavefield decomposition approaches may help solve related problems, such as the angle domain wavefield decomposition approach.

We conduct a test to compare the inversion results of conventional EI and DEI. In a common inversion situation (weak perturbation), conventional EI can provide a better long-wavelength gradient than FWI thanks to the artificial low-frequency components in the envelope data residuals. We can see many successful tests on the Marmousi model, which is a typical weak perturbation model for the FWI test. For strong scattering cases, because of the waveform Fréchet derivative, both EI and FWI cannot solve the problem of large-scale and strong perturbation inversion. This is already explained in detail by $[14,19]$. To further compare the results of EI and DEI, we provide the conventional EI and EI + FWI results in Figure 12. We can see that the conventional EI result (Figure 12a) is better than the conventional FWI result (Figure 6a) in salt body reconstruction, but can still only recover the information on the top boundary of the salt. The final EI + FWI result (Figure 12b) is similar to the conventional FWI result (Figure 6a), which demonstrates that the conventional EI method cannot completely solve the problems of FWI in strong scattering cases.
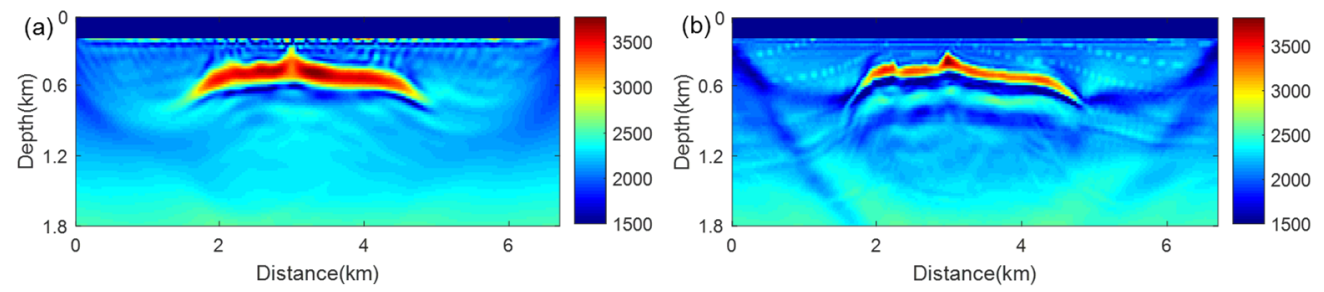

Figure 12. Conventional EI results. (a) Conventional EI result. (b) Conventional EI + FWI result.

A comparison of the computational costs between the DEI, WDDEI, and WDDEI + SGPD methods is shown in Table 1. The computation was conducted on a server with an Intel(R) Xeon(R) CPU E5-2690 v4 @ 2.60 GHz. The computational costs of WDDEI and 
WDDEI + SGPD are higher than that of DEI but are acceptable. Although the results of Figures $6 \mathrm{~b}$ and $7 \mathrm{a}$ seem similar, their contributions to the final inversion results are different. From the difference profiles of Figures $6 e$ and 7d, we know that the WDDEI + FWI method can provide more accurate velocity, both inside the salt body and in the subsalt area. This is very important for successive imaging and interpretation. We have conducted some tests and found that only by increasing the iteration time, the DEI result (Figure 13a) cannot approach the WDDEI result (Figure 7a), and the DEI + SGPD result (Figure 13b) cannot compensate the nonuniformity of the salt velocity. Considering the calculation efficiency and inversion effects, the proposed workflow has great potential to improve the quality of salt building.

Table 1. Computational costs comparison between DEI, WDDEI and WDDEI + SGPD.

\begin{tabular}{cc}
\hline Algorithm & Computational Time (50 Iterations) \\
\hline DEI & $50 \times 92 \mathrm{~s}$ \\
\hline WDDEI & $50 \times 540 \mathrm{~s}$ \\
\hline WDDEI + SGPD & $50 \times 540 \mathrm{~s}+92 \mathrm{~s}$ \\
\hline
\end{tabular}
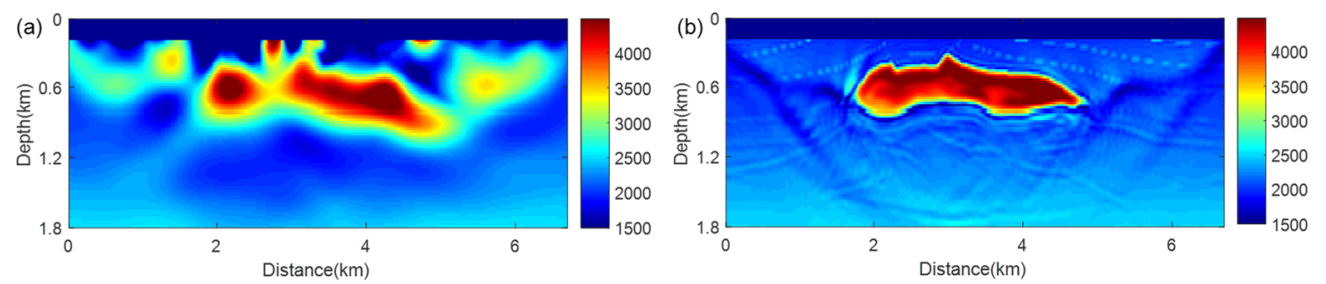

Figure 13. Further discussion about the DEI result. (a) DEI result with 10 more iterations than Figure 7a. (b) SGPD + FWI result based on Figure 6d.

There are some other factors we need to consider when we apply this method to real data [27]. Although the proposed methods show good antinoise performance, we still need to conduct denoising in real applications, because we may meet complex types of noise in real cases. The frequency bands in the multiscale DEI and FWI strategy should be carefully chosen according to the effective frequency band of the observed data and the scale of the inverted targets. We also need accurate source wavelet estimation to ensure a stable convergence. The source-independent strategy may also help to solve source-related problems. For improvement of calculation efficiency, we can introduce some high-efficiency wavefield decomposition algorithms or adopt GPU calculation.

The envelope field can be calculated more accurately by using the wavefield decomposition method, which can mitigate the effects of wavefield overlapping. Based on this, we propose a WDDEI method, which can build large and strong scattering salt structures in the absence of low-frequency data. To better invert the subsalt structures, we propose a structure-guided perturbation decomposition strategy. RTM images using the WDDEI + FWI velocity model can illustrate the shape of the salt body to guide the perturbation decomposition process. By combining the WDDEI and RTM-guided perturbation decomposition approach, both large-scale salt and some subsalt structures can be reconstructed effectively. The proposed method can recover relatively accurate salt structures and background structures with a moderate noise level.

Author Contributions: Conceptualization, P.Z.; methodology, P.Z., L.H.; software, P.Z., F.Z.; validation, P.Z., F.Z. and Q.F.; formal analysis, X.C.; resources, X.C.; data curation, Q.F.; writing-original draft preparation, P.Z.; writing—review and editing, P.Z., L.H.; visualization, F.Z.; supervision, P.Z.; project administration, P.Z., L.H., F.Z., X.C.; funding acquisition, P.Z. All authors have read and agreed to the published version of the manuscript. 
Funding: This research was funded by the National Natural Science Foundation of China (No. 42004106, No. 42130805, No. 42074154, No. 41974138), the open research project of the Key Laboratory of Deep-Earth Dynamics of Ministry of Natural Resources (J1901, J1901-16), the Natural Science Foundation of Jilin Province (No. YDZJ202101ZYTS020), the "Thirteenth Five-Year Plan" Science and Technology Project of Education Department of Jilin Province (No. JJKH20201001KJ), the Lift Project for Young Science and Technology Talents of Jilin Province (No. QT202116), and the Natural Science Foundation of Hebei Province (No. D2019403082).

Data Availability Statement: The data associated with this paper are available by contacting the corresponding author.

Acknowledgments: We would like to thank the Academic Editor Stan Mazur and the two anonymous reviewers for their insightful suggestions and contributions to this paper. P.Z. would like to thank Xiaobi Xie of University of California at Santa Cruz for discussions during the research.

Conflicts of Interest: The authors declare no conflict of interest.

\section{References}

1. Virieux, J.; Operto, S. An overview of full-waveform inversion in exploration geophysics. Geophysics 2009, 74, WCC127-WCC152. [CrossRef]

2. Shin, C.; Cha, Y.H. Waveform inversion in the Laplace domain. Geophys. J. Int. 2008, 173, 922-931. [CrossRef]

3. Shin, C.; Cha, Y.H. Waveform inversion in the Laplace-Fourier domain. Geophys. J. Int. 2009, 177, 1067-1079. [CrossRef]

4. Lewis, W.; Starr, B.; Vigh, D. A level set approach to salt geometry inversion in full-waveform inversion. In 82nd SEG Annual Meeting; Expanded Abstracts; Society of Exploration Geophysicists: Tulsa, OK, USA, 2012.

5. Kadu, A.; van Leeuwen, T.; Mulder, W. Salt reconstruction in full-waveform inversion with a parametric level-set method. IEEE Trans. Comput. Imaging 2017, 3, 305-315. [CrossRef]

6. Esser, E.; Guasch, L.; Herrmann, F.J.; Warner, M. Constrained waveform inversion for automatic salt flooding. Lead. Edge 2016, 35, 235-239. [CrossRef]

7. Peters, B.; Herrmann, F.J. Constraints versus penalties for edge-preserving full-waveform inversion. Lead. Edge 2017, 36, 94-100. [CrossRef]

8. Yong, P.; Liao, W.; Huang, J.; Li, Z. Total variation regularization for seismic waveform inversion using an adaptive primal dual hybrid gradient method. Inverse Probl. 2018, 34, 045006. [CrossRef]

9. Wang, G.; Yuan, S.; Wang, S. Retrieving low-wavenumber information in FWI: An efficient solution for cycle skipping. IEEE Geosci. Remote Sens. Lett. 2019, 16, 1125-1129. [CrossRef]

10. Chen, S.; Chen, G. Full waveform inversion based on time-integral-damping wavefield. J. Appl. Geophys. 2019, 163, 84-95. [CrossRef]

11. Yang, F.; Ma, J. Deep-learning inversion: A next-generation seismic velocity model building method. Geophysics 2019, 84, R583-R599. [CrossRef]

12. Zhang, Z.; Lin, Y. Data-driven seismic waveform inversion: A study on the robustness and generalization. IEEE Trans. Geosci. Remote Sens. 2020, 58, 6900-6913. [CrossRef]

13. Wu, R.S.; Chen, G. New Fréchet derivative for envelope data and multi-scale envelope inversion. In 79th EAGE Annual Meeting; European Association of Geoscientists \& Engineers: Paris, France, 2017.

14. Wu, R.S.; Chen, G. Multi-scale seismic envelope inversion using a direct envelope Fréchet derivative for strong-nonlinear full waveform inversion. arXiv 2018, arXiv:1808.05275. Available online: https://arxiv.org/abs/1808.05275 (accessed on 15 August 2018).

15. Bozdă̆, E.; Trampert, J.; Tromp, J. Misfit functions for full waveform inversion based on instantaneous phase and envelope measurements. Geophys. J. Int. 2011, 185, 845-870. [CrossRef]

16. Wu, R.S.; Luo, J.; Wu, B. Seismic envelope inversion and modulation signal model. Geophysics 2014, 79, WA13-WA24. [CrossRef]

17. Zhang, P.; Wu, R.S.; Han, L. Seismic envelope inversion based on hybrid scale separation for data with strong noises. Pure Appl. Geophys. 2019, 176, 165-188. [CrossRef]

18. Chen, G.; Wu, R.S.; Chen, S. Reflection multi-scale envelope inversion. Geophys. Prospect. 2018, 66, 1258-1271. [CrossRef]

19. Zhang, P.; Wu, R.S.; Han, L. Source-independent seismic envelope inversion based on the direct envelope Fréchet derivative. Geophysics 2018, 83, R581-R595. [CrossRef]

20. Chen, G.; Yang, W.; Chen, S.; Liu, W.; Gu, Z. Application of envelope in salt structure velocity building: From objective function construction to the full-band seismic data reconstruction. IEEE Trans. Geosci. Remote Sens. 2020, 58, 6594-6608. [CrossRef]

21. Chen, G.; Wu, R.S.; Chen, S. Multiscale direct envelope inversion: Algorithm and methodology for application to the salt structure inversion. Earth Space Sci. 2019, 6, 174-190. [CrossRef]

22. Hu, Y.; Wu, R.-S.; Han, L.-G.; Zhang, P. Joint multiscale direct envelope inversion of phase and amplitude in the time-frequency domain. IEEE Trans. Geosci. Remote Sens. 2019, 57, 5108-5120. [CrossRef] 
23. Luo, J.; Wu, R.S.; Chen, G. Angle domain direct envelope inversion method for strong scattering velocity and density estimation. IEEE Geosci. Remote Sens. Lett. 2020, 17, 1508-1512. [CrossRef]

24. Tarantola, A. Inversion of seismic reflection data in the acoustic approximation. Geophysics 1984, 49, 1259-1266. [CrossRef]

25. Liu, F.; Zhang, G.; Morton, S.; Leveille, J.P. An effective imaging condition for reverse-time migration using wavefield decomposition. Geophysics 2011, 76, S29-S39. [CrossRef]

26. Plessix, R.E. A review of the adjoint-state method for computing the gradient of a functional with geophysical applications. Geophys. J. Int. 2006, 167, 495-503. [CrossRef]

27. Zhang, P.; Gao, R.; Han, L.; Lu, Z. Refraction waves full waveform inversion of deep reflection seismic profiles in the central part of Lhasa Terrane. Tectonophysics 2021, 803, 228761. [CrossRef] 